\title{
Empleo de la gamificación en un curso de Fundamentos de Biología
}

\author{
Use of Gamification in Fundamentals of Biology Course
}

\section{Uso da gamificaçao em um curso de Fundamentos de Biologia}

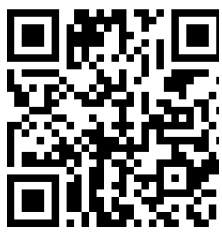

Corregido • Revised • Revisado: 16 / 10 / 2018

Aceptado • Accepted • Aprovado: 22/ 02 / 2019

\begin{abstract}
Resumen: Actualmente, en el Instituto Parauniversitario Plerus se observan limitaciones por parte de estudiantes tales como estudiar a última hora, no utilizar herramientas de referencia como libros de texto o artículos, y estudiar por una calificación y no por el conocimiento mismo. Por ello, el objetivo de esta investigación fue reforzar los contenidos del curso, el estudio continuo y la autoevaluación de los conocimientos adquiridos mediante el desarrollo de tareas gamificadas para el favorecimiento del aprendizaje del estudiantado de este curso. Para el desarrollo de la estrategia didáctica, participaron 29 estudiantes del curso Fundamentos de Biología (24 de los cuales concluyeron el curso y cinco lo retiraron) del Instituto Parauniversitario Plerus. Para este grupo, se estableció la confección de un portafolios y de actividades de reforzamiento (modelos de plasticina, experimentos caseros, sopas de letras, crucigramas, entre otros), cuya realización brindó recompensas mediante el obsequio a cada estudiante de calcomanías. Estas mismas fueron coleccionadas y cambiadas por premios didácticos, como un porcentaje en un examen corto. También se desarrolló un juego llamado Biología cr en la aplicación móvil Reinos ${ }^{\oplus}$. En él, cada persona elaboró preguntas de selección única que fueron revisadas por la docente $y$, seguidamente, se incorporaron para que fueran respondidas por otros compañeros y compañeras del grupo. Para determinar los resultados, se revisaron las calificaciones obtenidas por el grupo de estudiantes presentes a lo largo de todo el curso de Fundamentos de Biología, y se realizó una encuesta, que cada estudiante completó una vez concluida la utilización de esta estrategia. Para el análisis de los datos, se realizó un análisis sociodemográfico para la edad y el sexo, y un análisis descriptivo con distribución de frecuencias para el resto de preguntas. Desde la perspectiva docente, se obtuvieron buenos resultados, al darse una aprobación del $66,7 \%$ del
\end{abstract}


estudiantado. En cuanto a la perspectiva estudiantil, hubo aspectos positivos (mejoramiento de la comprensión de los temas, obligación a estar retomando de manera constante los temas vistos en clase y entenderlos por cuenta propia, incluyendo investigación personal, en caso de ser necesario) y negativos (incremento en la carga académica, preferencia del uso de estrategias didácticas tradicionales y falta de delimitación sobre lo más relevante de cada uno de los temas), los cuales deben ser considerados para futuras experiencias.

Palabras claves: Gamificación; juego; colaboración; aprendizaje significativo.

Abstract: Currently, at the Instituto Parauniversitario Plerus, there are limitations on the part of students such as studying at the last minute, not using reference tools such as textbooks or articles, and studying for a grade and not for the knowledge itself. Therefore, the objective of this research was to reinforce the course contents, the continuous study, and the self-evaluation of the acquired knowledge through the development of gammification tasks to favor the learning of the students of this course. For the development of the didactic strategy, 29 students from Fundamentals of Biology course, at the Instituto Parauniversitario Plerus, took part: 24 of whom completed the course and five withdrew it. For this group, a portfolio and reinforcement activities (plasticine models, homemade experiments, word search puzzles, crossword puzzles, among others) were established. The realization of these activities provided rewards through the gift of stickers to each student. These rewards were collected and exchanged for didactic prizes, such as a percentage in a short exam. A game called Biology cr was also developed in the Reinos ${ }^{\circledR}$ mobile application. In it, each student developed unique selection questions that were reviewed by the teacher and then incorporated to be answered by other classmates. To determine the results, the grades obtained by the students enrolled in the Fundamentals of Biology course were reviewed, and a survey was carried out, which each student completed once the implementation of this strategy was concluded. To analyse the data, a sociodemographic analysis was carried out for age and sex, and a descriptive analysis with frequency distribution was carried out for the rest of the questions. From a teaching perspective, good results were obtained, with $66.7 \%$ of the student body passing. With regard to the student perspective, there were positive aspects (improvement in the understanding of subjects, obligation to constantly take up the subjects studied in class and understand them on their own, including personal research, if necessary) and negative aspects (academic load increase, preference for the use of traditional didactic strategies and lack of delimitation of the most relevant aspects of each of the subjects), which must be considered for future experiences.

Keywords: Gamification, game, collaboration, significant learning.

Resumo: Atualmente, no Instituto Parauniversitario Plerus observam-se limitações por parte de estudantes tais como estudar a última hora, não utilizar ferramentas de referência como livros de texto ou artigos, e estudar por uma qualificação e não pelo conhecimento em sí. Por isso, o objetivo desta pesquisa foi reforçar os conteúdos do curso, o estudo contínuo e a auto-avaliação dos conhecimentos adquiridos através do desenvolvimento de tarefas gamificadas para favorecer a aprendizagem dos estudantes deste curso. Para o desenvolvimento da estratégia didática, participaram 29 estudantes do curso Fundamentos de Biologia (24 dos quais concluíram o curso e cinco o retiraram) do Instituto Parauniversitario Plerus. Para este grupo, estabeleceu-se um portfólio e fortalecimento das atividades 
(modelos de plasticina, experimentos em casa, quebra-cabeças, palavras cruzadas, entre outros), cuja realização brindou recompensas mediante o obsequio a cada estudante de decalques. Estas mesmas foram colecionadas e mudadas por prêmios didáticos, como uma percentagem em um exame curto. Também se desenvolveu um jogo chamado Biologia cr na aplicação móvel Reinos ${ }^{\circledast}$. No jogo, cada pessoa elaborou perguntas de seleção única que foram revisadas pela docente e, seguidamente, se incorporaram para que fossem respondidas por outros colegas do grupo. Para determinar os resultados, foi realizada uma análise sociodemográfica relacionada com a idade e sexo e uma análise descritiva com distribuição de frequência para o restante das questões. Desde a perspectiva docente, obtiveram-se bons resultados, ao dar-se uma aprovação de $66,7 \%$ dos alunos. Quanto à perspectiva do estudante, teve aspectos positivos (melhoria do entendimento dos temas, obrigação a estar retomando de maneira constante os temas vistos em classe e entendê-los por conta própria, incluindo investigação pessoal, em caso de ser necessário) e negativos (aumento da carga acadêmica, preferência pelo uso de estratégias didáticas tradicionais e falta de delimitação dos aspectos mais relevantes de cada um dos assuntos), que devem ser considerados para experiências futuras.

Palavras-chave: Gamificação; jogo; colaboração; aprendizagem significativa.

\section{Introducción}

Actualmente, en el Instituto Parauniversitario Plerus se observan limitaciones por parte de gran cantidad de estudiantes, entre ellas: estudiar a última hora, no utilizar herramientas de referencia como libros de texto o artículos, y estudiar por una calificación y no por el conocimiento mismo.

Para poder solventar esta situación y ayudar al aprendiente en su proceso de autorregulación, se planteó para el curso Fundamentos de Biología (DM-102) la utilización de una experiencia didáctica de gamificación. De esta manera, el objetivo de esta investigación fue reforzar los contenidos del curso, el estudio continuo y la autoevaluación de los conocimientos adquiridos mediante el desarrollo de tareas gamificadas para el favorecimiento del aprendizaje de los estudiantes y las estudiantes de este curso.

\section{Referentes conceptuales y antecedentes de la estrategia didáctica}

\section{Antecedentes}

La gamificación es una nueva tendencia, en donde elementos de juegos son usados en contextos poco comunes, como educación, salud, áreas sociales y mercadeo (Turan, Avinc, Kara y Goktas, 2016). El término fue acuñado por Nick Pelling en 2002 (Kim, 2015), pero se dice que su primer uso se dio en 2008 (Fleischmann y Ariel, 2016) y su adopción no se extendió sino hasta después de la segunda mitad de 2010 (Dicheva, Dichev, Agre y Angelova, 2015). 
doi: http://dx.doi.org/10.15359/ree.23-2.10

URL: http://www.una.ac.cr/educare

CORREO: educare@una.cr

Sin embargo, su uso en educación ha sido discutido ampliamente antes de la aparición de los juegos computacionales. Según Emel'yanenko, Vetoshko, Malinnikov, Malashenko y Vetoshko (2016), los juegos usados en la Unión Soviética (siglo XX) y en los Estados Unidos (siglo XXI) fueron los precursores de la gamificación. Nelson (2012) menciona que en la Unión Soviética estos juegos fueron empleados para motivar a los grupos de trabajadores, sin depender de los incentivos monetarios del estilo capitalista. Asimismo, Lenin propuso la teoría de la competencia socialista en la que se promovía esta actitud entre la clase trabajadora, grupos de esta o fábricas para motivar una mayor producción. Posteriormente, en los Estados Unidos se desarrolló una tendencia de diversión en el trabajo. Esta consistía en imaginar dicho lugar como un sitio divertido y lúdico en lugar de uno de trabajo y pesadez, generando la visión de verlo como un juego que incluía la presencia de elementos, los cuales se explicarán más adelante. Es a partir de estas experiencias que se desarrolla la gamificación y sus características.

\section{Definición}

La gamificación es una estrategia didáctica que se basa en el concepto de emplear mecánicas y técnicas del diseño de juegos para atraer y motivar a las personas a alcanzar sus objetivos (Barlow y Fleming, 2016), en contextos que no lo son (Olsson, Mozelius y Collin, 2015).

Fleischmann y Ariel (2016) mencionan que la definición tiene tres componentes:

1. El uso de atributos del juego: incluye dinámicas, principios del diseño, psicología, viaje de cada persona jugadora, guiones y narración o cualesquiera otros aspectos de juegos.

2. Conducción del comportamiento de la persona:como motivación, interacción, adicción, competición, colaboración, conciencia, aprendizaje, o cualquier otro comportamiento observado durante su realización.

3. Desarrollo en un contexto que no involucra juegos: puede ser cualquier otro diferente como educación, trabajo, salud y belleza, participación en la comunidad, compromiso cívico, voluntariado, etc.

El concepto es diferente de aquel de un juego educacional o serio. Mientras que el último describe el diseño de juegos completos para propósitos que no son de entretenimiento, las aplicaciones gamificadas emplean meramente elementos de estos mismos (Dicheva et al., 2015). Como el término sugiere, la gamificación no es crear un juego, sino transferir algunas de sus características positivas a algo que no lo es (Kim, 2015). 


\section{Características de la gamificación en la educación}

La gamificación como estrategia didáctica está diseñada para balancear la información de un determinado curso con jugar, y la habilidad de sus participantes de retener y aplicar la materia de este en el mundo real, en un ambiente de aprendizaje basado en tomar acciones, experimentar consecuencias y trabajar por objetivos. Además, permite cometer errores a través de la experimentación en un ambiente libre de riesgos (Abersek, 2016).

Junto con ello, es apreciada como un medio para atraer al estudiantado a adquirir conocimientos en distintos cursos académicos y a profundizar en estos (Barlow y Fleming, 2016), al presentar las características de desafío, curiosidad y fantasía (Fan, Xiao y Su, 2015). Esto se debe a que dentro del contexto de los juegos, las personas voluntariamente invierten horas incontables en el desarrollo de habilidades para la resolución de problemas, para nivelarse y para alcanzar sus metas finales (Chen, Burton, Mihaela y Whittinghill, 2015). Con ello, se busca motivar y comprometer al estudiantado por períodos prolongados, y emplear ese mismo nivel de motivación y compromiso en otros contextos distintos donde no se utilice esta estrategia (Cheong, Filippou y Cheong, 2014).

El elemento de gamificación es derivado del marco de referencia de mecánicas (relacionadas con juegos que describen componentes específicos de este en el nivel de representación de la información y algoritmos), dinámicas (actuación del comportamiento de la mecánica del tiempo de ejecución en las entradas de la persona y las otras salidas a través del tiempo) y estéticas (respuesta emocional deseada por la persona cuando interactúa con el sistema de juego) (MDA, por sus siglas en inglés), que se basa en la teoría del diseño de juegos (Hamzah, Ali, Saman, Yusoff y Yacob, 2015).

Adicionando elementos de juegos a actividades que no los involucran introduce emoción y creatividad a lo que se está haciendo. La gamificación trabaja para incrementar la competitividad natural y ofrece recompensas para seguir jugando, tales como puntos, medallas, niveles, tablas de clasificación y desafíos para aumentar la motivación. Todo esto significa cierto grado de logro y ayuda a motivar a cada estudiante para continuar participando (Fisher, Beedle y Rouse, 2014).

Daley inclusive menciona una lista de las cosas que genera la diversión. La misma incluye: opciones y control del jugador o de la jugadora, recompensas, conocimientos, niveles alcanzables, puntos por experiencias, gratificaciones y potenciadores, misiones desafiantes, sorpresas, narrativas convincentes y personajes fantásticos. Estas categorías no están en cada juego y cada una no tiene que estar presente para hacerlo bueno (Fisher et al., 2014).

Existen distintos elementos a considerar para la adecuada implementación de la gamificación en el proceso de enseñanza-aprendizaje (Cheong et al., 2014) como por ejemplo, entender a las personas que participarán en la estrategia didáctica. 
doi: http://dx.doi.org/10.15359/ree.23-2.10

URL: http://www.una.ac.cr/educare

CORREO: educare@una.cr

\section{Metodología}

Esta investigación siguió un enfoque cuantitativo y cualitativo, a través del empleo de herramientas que permitieran conocer los resultados académicos del estudiantado del curso DM-102, y sus opiniones acerca de esta experiencia gamificada.

Para el desarrollo de dicha investigación, participaron 29 estudiantes del curso Fundamentos de Biología (24 concluyeron el curso y cinco lo retiraron) del Instituto Parauniversitario Plerus, asociado a la Universidad Libre de Costa Rica, el cual constó de dos horas semanales en el segundo cuatrimestre de 2016. Para este grupo, se estableció la confección de un portafolios. Estuvo dividido por temas y para cada uno se solicitó un resumen, un cuestionario con preguntas relevantes (contestadas en casa por cada persona y revisadas en clases por la docente) y una actividad de reforzamiento (modelos en plastilina, experimentos caseros, sopas de letras, crucigramas y actividades de movimiento como la representación de un conocimiento a través de una dramatización). A las personas que realizaron estos trabajos se les otorgaron recompensas, mediante el obsequio de calcomanías. Estas fueron coleccionadas y cambiadas por premios didácticos, como un porcentaje en un examen corto.

Otra actividad realizada fue brindar al estudiantado materiales de apoyo tales como documentales y artículos de periódicos. Con ello, se desarrolló un canal llamado Biología cr en la aplicación móvil Reinos ${ }^{\oplus}$. En él, cada persona elaboró preguntas de selección única que fueron revisadas por la docente y, seguidamente, se incorporaron para que fueran respondidas por otros compañeros y compañeras del grupo. De esta manera, cada persona colaboró en el aprendizaje del resto del grupo y permitió que la docente observara si los objetivos de aprendizaje de los distintos temas fueron asimilados, pues la aplicación brindó los puntajes de cada estudiante para los distintos juegos en los que participó y una tabla de clasificación con el puntaje acumulado. Este mismo incluyó las preguntas contestadas correctamente y las elaboradas para el canal.

Para determinar los resultados de la estrategia didáctica empleando la gamificación, se revisaron las calificaciones obtenidas por el grupo de estudiantes presentes a lo largo de todo el curso, donde se implementó dicha estrategia, específicamente exámenes cortos, portafolios, examen parcial y examen final. Asimismo, se realizó una encuesta, más específicamente un cuestionario, que cada estudiante completó una vez concluida la utilización de esta estrategia. Esta misma ya fue empleada previamente para recopilar información sobre la implementación de otra estrategia didáctica (Mora-Román, 2014).

Para el análisis de los datos, se realizó un análisis sociodemográfico para la edad y el sexo indicados por las personas, y un análisis descriptivo con distribución de frecuencias para el resto de preguntas abiertas presentes en ella. Para asegurar la validez de los datos y los resultados, las notas obtenidas fueron mantenidas en un registro al cual únicamente la docente del curso tuvo acceso, y en cuanto a las encuestas, fueron contestadas de forma anónima y voluntaria por parte del estudiantado que asistió el día en que se aplicó.

6

Los artículos de la Revista Electrónica Educare del Centro de Investigación y Docencia en Educación de la Universidad Nacional, Costa Rica, se comparten bajo términos de la Licencia Creative Commons: Reconocimiento, No Comercial, Sin Obra Derivada 3.0 Costa Rica. Las autorizaciones adicionales a las aquí delimitadas se pueden obtener en el correo: educare@una.cr 


\section{Resultados, análisis y discusión}

En primer lugar, se hizo una revisión de las calificaciones obtenidas por el total de estudiantes que realizaron todas las evaluaciones del curso. Las calificaciones se calcularon con base a 100. Los resultados obtenidos se aprecian en la Tabla 1:

Tabla 1: Calificaciones finales del estudiantado que completó el curso Fundamentos de Biología (DM102) en el Instituto Parauniversitario Plerus durante el segundo cuatrimestre de 2016

\begin{tabular}{ccc}
\hline Calificación final & Número de estudiantes & Porcentaje (\%) \\
\hline Menos de 60 & 2 & 8,3 \\
60 a 69 & 6 & 25,0 \\
70 a 79 & 14 & 58,3 \\
80 a 89 & 1 & 4,2 \\
Más de 90 & 1 & 4,2 \\
\hline TOTAL & 24 & 100,0 \\
\hline
\end{tabular}

Nota: Elaboración propia.

Estos valores muestran una aprobación por parte del 66,7\% de los estudiantes y las estudiantes, lo cual representa una gran parte del grupo que cumplió con todas las evaluaciones. Este resultado global obtenido muestra un primer indicio sobre los aspectos positivos de la gamificación en el proceso de enseñanza, debido a la influencia que tiene en los procesos de enseñanza y de aprendizaje en lo que respecta a motivación, disfrute y compromiso para llevar a cabo los trabajos asignados (Piñeiro-Otero y Costa-Sánchez, 2015). No obstante, para poder verificar esto, se llevó a cabo la evaluación de la labor realizada y, de este modo, conocer la opinión del estudiantado.

Por ende, se resumió la información de los 19 cuestionarios que fueron debidamente completados. De acuerdo con la Tabla 2, la distribución de estudiantes por edad fue la siguiente:

Tabla 2: Distribución de estudiantes en el curso Fundamentos de Biología (DM-102) en el Instituto Parauniversitario Plerus durante el segundo cuatrimestre de 2016 de acuerdo con su edad en años

\begin{tabular}{ccc}
\hline Edad (años) & Número de estudiantes & Porcentaje (\%) \\
\hline Menos de 20 & 1 & 5,3 \\
20 a 30 & 14 & 73,7 \\
30 a 40 & 2 & 10,5 \\
Más de 40 & 1 & 5,3 \\
No responde & 1 & 5,3 \\
\hline TOTAL & 19 & 100,0 \\
\hline
\end{tabular}

Nota: Elaboración propia. 
doi: http://dx.doi.org/10.15359/ree.23-2.10

URL: http://www.una.ac.cr/educare

CORREO: educare@una.cr

Esta información señala que la población fue sumamente heterogénea, al encontrarse en edades que oscilaron entre 19 y 44 años. Lo anterior permite comprender que la aplicación de esta estrategia didáctica tuvo diferentes visiones, de acuerdo con los años con los que cada persona cuenta desde su egreso de la educación secundaria o su ingreso a la universidad. Además, en ocasiones implica que al aumentar la edad, la persona tiene mayor probabilidad de haber sido educada empleando el modelo tradicional. Esta educación basada en el hecho de percibir a la persona como receptora de información (Rodríguez Cavazos, 2013) dificulta el empleo de estrategias didácticas contemporáneas, lo cual debe ser diagnosticado antes de ponerlas en práctica.

En lo que respecta a la distribución de acuerdo con el sexo, se exhibió que el grupo que completó la encuesta se encontraba conformado por ocho personas de sexo masculino y 11 de femenino, equivalente a un 42,1 y un $57,9 \%$, respectivamente. En este caso, se contó con una muestra en donde no existía una diferencia significativa entre personas de sexo femenino en comparación con las personas de sexo masculino.

Después, se hizo la consulta acerca de si habían utilizado la gamificación en un curso anteriormente. Del total de respuestas, el $68,4 \%$ de personas expresó que jamás había usado la gamificación previamente. En contraposición, el 31,6\% respondió que sí la había empleado durante su experiencia colegial $(15,8 \%)$ o universitaria $(15,8 \%)$. Cabe destacar que las personas que respondieron afirmativamente a esta interrogante tenían una edad entre 24 y 44 años, lo cual reafirma que el fundamento de esta estrategia no es nuevo, sino que lo novedoso es el concepto como tal (Dicheva et al., 2015; Fleischmann y Ariel, 2016). Además, como la gran mayoría del grupo no ha entrado en contacto con esta, es posible apuntar a su implementación como una herramienta interesante de analizar para el proceso de enseñanza-aprendizaje parauniversitario.

A continuación, se quiso saber si la implementación de esta estrategia didáctica fue útil para mejorar el rendimiento académico o el proceso de enseñanza-aprendizaje asociado al curso. El 63,2\% contestó que le sirvió el uso de la gamificación para obtener mejores resultados en el desarrollo de la asignatura. La siguiente pregunta, relacionada con algún comentario positivo sobre el porqué fue útil la gamificación, permitió observar una gama de beneficios derivados de su implementación. Un estudiante indicó que el uso de esta estrategia ayudó a "una mayor comprensión de los temas", mientras que otros tres señalaron que "permitió recordar más fácilmente la materia impartida de ese modo" y a la vez "aprender más rápidamente." Otros comentarios de esta índole apuntan a que con las actividades se aprende de una mejor manera y obliga a la persona a tener que estar retomando constantemente los temas para la realización de las actividades. Asimismo, además de tener una clase más entusiasmada, el estudiantado puede inspirarse para realizar investigaciones exteriores y generar un interés creciente en el curso (Fisher et al., 2014). 
De todos los comentarios favorables, llama la atención que a dos personas les permitió ir más allá del aula propiamente. Ellas mismas expresan cómo la estrategia didáctica las ayudó a analizar la materia vista en clase y a tratar de entenderla por cuenta propia, así como que les dio la motivación para investigar por sí mismas sobre los contenidos vistos en clase. Como lo indican Chen et al. (2015), Leblanc apunta que la gamificación puede motivar a las personas a participar más profundamente y cambiar su concepto de sí mismas como estudiantes.

No obstante, el 36,8\% señaló que al contrario de la mayoría, la gamificación no representó una experiencia positiva dentro del curso. Comentarios no favorables sobre la estrategia que se leyeron se relacionaron mucho con la carga académica que demanda el curso al emplearse el desarrollo de juegos para llevar a cabo el proceso de enseñanza-aprendizaje. Al respecto, un estudiante expresa "son demasiados trabajos, no da tiempo para las otras materias." Como complemento, se indica que en algunos casos el estudiantado prefiere emplear los métodos tradicionales de aprendizaje en vez de técnicas innovadoras. Esto se aprecia mediante los comentarios de dos estudiantes, quienes escribieron lo siguiente: "A mí me parece que es mejor estudiar la materia que hacer resúmenes, los cuales no ayudan y se hacen por presentarlos" y "pasé mucho tiempo haciendo trabajos que pude haber utilizado para estudiar". Como lo indica Moreno Olivos (2011), la escuela se sigue apegando al peso de las tradiciones pedagógicas. Ante esto, se hace difícil la aplicación de estrategias de vanguardia en el ámbito parauniversitario. Por ello, tal vez mejorar el acercamiento de la población estudiantil hacia esta y otras estrategias implica la necesidad de que el personal docente debe tomar un tiempo para explicar su fundamento y sus características, con el propósito de que las personas que la utilizarán comprendan la razón de efectuar una u otra actividad.

Otro detalle particular es el enfocar la gamificación hacia determinados objetivos del curso y no abusar de esta. Lo anterior considerando que un estudiante expresó que la gamificación no brindó una delimitación de la materia más importante. Esta es una de las desventajas mencionadas para esta estrategia, pues existen casos donde los juegos no motivan a aprender, sino únicamente a jugar. El estudiantado sigue preocupándose, entonces, por obtener buenas notas, pero no la educación propia y los materiales educativos, e inclusive se llega a desilusionar de las técnicas del juego y del aprendizaje en general (Emel'yanenko et al., 2016).

Otro aspecto que está relacionado más con la asignatura que con la estrategia didáctica son los contenidos del curso propiamente, pues se encontraron cuatro comentarios negativos en relación con ello. Dos fueron: "La cantidad de materia excesiva hace difícil comprender toda la materia en un corto perío do" y se "maneja mucha materia en tan poco tiempo que ocasiona que esta se acumule, más si se llevan otros cursos." Por ello, dentro de lo positivo se halló que es necesario hacer una revisión de los contenidos del temario, y así, determinar si debe darse más énfasis a unos en comparación con otros, junto con definir aquellos en los cuales la gamificación puede brindar experiencias más favorables en el señalamiento del orden de importancia de las ideas referentes a ellos. 
doi: http://dx.doi.org/10.15359/ree.23-2.10

URL: http://www.una.ac.cr/educare

CORREO: educare@una.cr

Por otro lado, se consultó acerca de cuáles fueron los aspectos que no resultaron del agrado del estudiantado respecto al uso de la gamificación como estrategia didáctica en el curso. Cinco estudiantes respondieron que el uso de esta estrategia implicó la inversión de mucho tiempo. De hecho, un estudiante escribió que "se necesita más tiempo para poder apropiarse de todos los conocimientos del curso". Por ello, para usos futuros es necesario considerar el emplearla en un número menor de actividades o el realizarlas en el aula.

Esta última sugerencia surge a partir de que otra de las limitaciones apreciadas por las personas fue el trabajo que implicaba el desarrollar las actividades fuera del aula. Esto fue expresado por siete estudiantes que completaron la encuesta facilitada. Lo anterior es justificado a partir de varios comentarios, uno de los cuales señala: "mucha materia y muchos trabajos para la casa." Asimismo, sería ideal evaluar los conocimientos que el estudiantado debe adquirir al finalizar el curso, pues se opinó que es "mucha materia y muchos trabajos que al final no fueron importantes" " "se debería ir al grano."

A pesar de esto, las limitaciones presentadas tienen la posibilidad de ser solventadas, evaluando la carga académica que representa para una persona el realizar las actividades planteadas y compararla con el tiempo tanto dentro como fuera del aula que se debe dedicar a la asignatura. Además, es relevante evaluar el grado de dedicación del estudiantado en general, pues como lo indica una estudiante: "me parece que debe ser un poco más llevadero para las personas que trabajamos y llevamos cuatro cursos más." Si bien es cierto que el uso de juegos puede generar un alto tiempo de involucramiento (Fleischmann yAriel, 2016), también es necesario evaluar la realidad del tiempo que las personas verdaderamente pueden dedicar a su educación, según factores sociales, familiares y económicos.

Otros aspectos desfavorables fueron acerca de escribir mucho y que los resúmenes no son del agrado para mejorar el proceso de enseñanza-aprendizaje. Tres estudiantes no indicaron molestia alguna con la estrategia.

Finalmente, en lo que respecta a si recomendarían el empleo de la estrategia en futuros cursos de la carrera, un 58\% respondió afirmativamente, mientras que el $42 \%$ indicó que no la utilizaría.

Las respuestas a favor de la gamificación mencionan que la estrategia es buena para incentivar el estudio y se mejora el proceso de aprendizaje. Referente a esto último una estudiante comentó:

Nos permite estudiar de una manera diferente, no solo estar leyendo y memorizando. Al jugar uno memoriza el juego y a la hora del examen el juego se le viene a la memoria. 
Un aspecto a destacar es que a pesar de la respuesta favorable sobre esta manera de mejorar el proceso de enseñanza-aprendizaje, tres respuestas reafirman la necesidad de delimitar en mayor medida el tiempo comprendido para llevar a cabo las actividades para que la gamificación pueda ser útil en los cursos futuros.

En contraposición, algunos de los comentarios desfavorables de su utilización fueron sobre un uso más delimitado, pues de la manera como se hizo en el curso se vuelve recargado y el hecho de que se deben establecer los objetivos de la estrategia a partir del perfil de salida del estudiantado al momento de aprobar el curso. Si las actividades son muy generales, pueden ser poco exitosas, como lo expresa una persona: "Deja muchas lagunas."

\section{Conclusiones}

El empleo de la gamificación como estrategia didáctica en un grupo de educación superior es innovador. Esto se reafirma con la encuesta realizada al estudiando, pues el 68,4\% de personas indicó que nunca la habían utilizado en ámbitos anteriores. Por ello, el documentar los resultados obtenidos presenta un panorama inicial sobre aspectos positivos y negativos acerca de su incorporación por parte del profesorado.

Desde la perspectiva docente, se obtuvieron buenos resultados, al darse una aprobación del curso Fundamentos de Biología del $66,7 \%$ de las personas. No obstante, no se puede estar del todo satisfecho, al ser necesario evaluar su uso junto con otras estrategias didácticas, para incrementar dicho porcentaje.

Por otra parte, más del $60 \%$ señaló que la implementación de la estrategia fue útil para incrementar su rendimiento académico o su proceso de enseñanza-aprendizaje asociado al curso, dado que ayudó a mejorar la comprensión de los temas, recordar más fácilmente la materia, obligar a estar retomando de manera constante los temas vistos en clase y tratar de entenderlos por cuenta propia, investigando en caso de ser necesario; se cumple, de esta forma, el reforzamiento de los contenidos vistos en clase.

En contraposición, algunas de las desventajas de la gamificación fueron un incremento en la carga académica, debido al desarrollo de juegos, el preferir el uso de estrategias didácticas tradicionales y la falta de una delimitación más clara acerca de la información más relevante de cada uno de los temas.

Por ello, en futuras experiencias el personal docente debe establecer una serie de medidas que mejoren su implementación, entre ellas, tomar un tiempo para explicar su fundamento y sus características, emplearla en un número menor de actividades o realizarlas en el aula, y evaluar la realidad del tiempo que las personas verdaderamente pueden dedicar a su educación, según factores sociales, familiares y económicos. También sería de provecho efectuar una experiencia con al menos dos grupos, uno en el cual se emplee la gamificación y en el otro únicamente estrategias didácticas tradicionales. 
doi: http://dx.doi.org/10.15359/ree.23-2.10

URL: http://www.una.ac.cr/educare

CORREO: educare@una.cr

\section{Referencias}

Aberšek, B. (2016). Schola ludus. Journal of Baltic Science Education, 15(5), 556-558. Recuperado de $\quad$ http://www.scientiasocialis.It/jbse/files/pdf/vol15/556-558.Abersek JBSE Vol.15 No.5.pdf

Barlow, T. y Fleming, B. (2016). A science classroom that's more than a game. Teaching Science: The Journal of the Australian Science Teachers Association, 62(2), 31-37.

Chen, Y., Burton, T., Mihaela, V. y Whittinghill, D. M. (2015). Cogent: A case study of meaningful gamification in education with virtual currency. iJET: International Journal of Emerging Technologies in Learning, 10(1), 39-45. doi: http://dx.doi.org/10.3991/ijet.v10i1.4247

Cheong, C., Filippou, J. y Cheong, F. (2014). Towards the gamificación of learning: Investigating student perceptions of game elements. Journal of Information Systems Education, 25(3), 233-244.

Dicheva, D., Dichev, C., Agre, G. y Angelova, G. (2015). Gamification in education: A systematic mapping study. Educational Technology and Society, 18(3), 75-88.

Emel'yanenko, V. D., Vetoshko, A. N., Malinnikov, S. G., Malashenko, I. V. y Vetoshko, L. I. (2016). Man's values and ideologies as a basis of gamification. International Journal of Environmental \& Science Education, 11(18), 12576-12592. Recuperado de http://www.ijese. net/makale/1728

Fan, K.-K., Xiao, P. y Su, C. (2015). The effects of learning styles and meaningful learning on the learning achievement of gamification health education curriculum. Eurasia Journal of Mathematics, Science and Technology Education, 11(5), 1211-1229. doi: https://doi. org/10.12973/eurasia.2015.1413a

Fisher, D. J., Beedle, J. y Rouse S. E. (2014). Gamification: A study of business teacher educators' knowledge of, attitudes toward, and experiences with the gamification of activities in the classroom. The Journal of Research in Business Education, 56(1), 1-16.

Fleischmann, K. y Ariel, E. (2016). Gamification in science education: Gamifying learning of microscopic processes in the laboratory. Contemporary Educational Technology, 7(2), 138159. Recuperado de https://files.eric.ed.gov/fulltext/EJ1106923.pdf

Hamzah, W. M. A. F. W, Ali, N. H., Saman, M. Y. M., Yusoff, M. H. y Yacob, A. (2015). Influence of gamification on students' motivation in using E-learning applications based on the motivational design Model. iJET: International Journal of Emerging Technologies in Learning, 10(2), 30-34. doi: http://dx.doi.org/10.3991/ijet.v10i1.4355 
Kim, B. (2015). The popularity of gamification in the mobile and social era. American Library Association, 51(2), 5-9. Recuperado de https://journals.ala.org/index.php/ltr/article/ view/5628/6944

Mora-Román, J. J. (2014). Implementación del aprendizaje colaborativo durante el Laboratorio de Cálculos Farmacéuticos Aplicados en la Facultad de Farmacia de la Universidad de Costa Rica. Revista Electrónica Educare, 18(2), 159-175. doi: http://dx.doi.org/10.15359/ree.18-2.8

Moreno Olivos,T.(2011).Didáctica en la educación superior: Nuevos desafíos en el sigloXXI. Revista Perspectiva Educacional, 50(2), 26-54. Recuperado de http://www.perspectivaeducacional. cl/index.php/peducacional/article/viewFile/45/24

Nelson, M. J. (2012). Soviet and American precursors to the gamification of work. En Proceedings of the 16th International Academic MindTrek Conference (pp. 23-26). Proceedings of the International Academic MindTrek Conference. doi: https://doi. org/10.1145/2393132.2393138

Olsson, M., Mozelius, P. y Collin, J. (2015). Visualisation and gamification of e-Learning and programming education. The Electronic Journal of e-Learning, 13(6), 441-454. Recuperado de https://files.eric.ed.gov/fulltext/EJ1087309.pdf

Piñeiro-Otero, T. y Costa-Sánchez, C. (2015). ARG (alternate reality games). Contributions, limitations, and potentialities to the service of the teaching at the university level. Comunicar, 22(44), 141-148. doi: http://dx.doi.org/10.3916/C44-2015-15

Rodríguez Cavazos, J. (2013). Una mirada a la pedagogía tradicional y humanista. Presencia Universitaria, 3(5), 36-45. Recuperado de http://eprints.uanl.mx/3681/1/Una mirada a la pedagog\%C3\%ADa tradicional y humanista.pdf

Turan, Z., Avinc, Z., Kara, K. y Goktas, Y. (2016). Gamification and education: Achievements cognitive loads, and views of students. iJET: International Journal of Emerging Technologies in Learning, 11(7), 64-69. doi: http://dx.doi.org/10.3991/ijet.v11i07.5455 